\title{
Meal Planning Tablet Software for Training of Registered Dietitians
}

\author{
Yasuteru Hosokawa, Naomi Suzuki, Keiji Ozaki, Keiko Takahashi and Sayaka Kawabata
}

\begin{abstract}
Many trial and error of a meal planning are need for training of registered dietitians. On the other hand, calculations of many items which are nutrient, food groups, ingredients, cooking method, and so on are needed for the evaluation of the meal planning. In this study, we report about a development of a meal planning tablet software for training of registered dietitians. This software can use easily and show the calculation result immediately. By using this software for training of registered dietitians, students have many trial and error experiences of a meal planning.
\end{abstract}

Keywords - Registered Dietitians, Meal Planning, Tablet PC, Trial and Error.

\section{INTRODUCTION}

Registered Dietitians plan daily meals in hospitals, schools, and so on. In the hospital, they plan meals for various kinds of patients. In the school, they plan school lunch for students. In these situations, there are three important points for planning daily meals. The planner has to plan different meals every day, to plan good combination of meals, and to plan suitable nutrient meals. If one of them is lacked, the meal cannot be accepted. For instance, the meal which has good taste, good combination, and suitable nutrients is supplied every day, everybody may be getting sick in a few days. As another example, curry rice and sashimi are not good combination, because we cannot taste sashimi which has simple taste and cold after eating curry rice which is complextaste and spicy. Registered dietitians plan daily meals with considering these points. However, there is no perfect answer for planning meals and they decide the planning meals by their experience and knowledge. Therefore, many trial and error of planning meals are needed for training for registered dietitians.

On the other hand, there are many evaluation items of a meal planning. A nutrient which has many items, a number and a kind of dishes, food groups, ingredients, cooking method and so on are measured for evaluations. These quantities are changed by patient's age, gender, activity amount, and diseases. Considering many data of meals, many types of patients, and so on are needed for evaluations of a meal planning.

In order to calculate the evaluation items of a meal planning, some applications for PC or Web have been provided [1]-[3]. By

Yasuteru Hosokawa, Naomi Suzuki, Keiji Ozaki, Sayaka Kawabata and Keiko Takahashi, Shikoku University, Japan using these software, the calculation can be carried out in a short time and many times. However, these softwares have problems for students. One is a cost. In many schools, expensive software for training of registered dieticians may be installed in computer rooms. The student can use only opened the room. Buying the software may be not easy for the student. Therefore, students don't have enough time for trial and error for a meal planning. This problemcan be solved by using web applications. However, these applications also have problems. Web applications work on an internet browser. Thus, the usability is limited by functions of the browser. Additionally, a connection to the internet is required. By developing a tablet software these problems can be solved.

In this study, a meal planning tablet software for training of registered dietitians is developed and revised by students of registered dietitians. In Sect. II, the tablet software is explained, In Sect. III, questionnaire results in the classroom is shown. In Sect. IV, discussions are descried. Finally, conclusions are described in Sect. V.

\section{MEAL PlanNing TABLET SOFTWARE}

\section{A.Development Environment and Data}

iPad [4] was selected as a main target for developing the software. The reason is easiness of the developing software. There are some operating systems for tablet PCs. The most famous and used operating system is Android [5]. Many kinds of hardware have been sold. Especially, many kinds of smart phones are sold. Therefore, the performance is also wide variety. It means to need to check the adaption of each hardware. In case of iPad, there are some versions. However, the performance of hardware is relativity stable. Additionally, IDE which is called as Xcode [6] and all emulators are provided by Apple Inc.. The additional reason is a large screen. A meal planning needs to calculate and to show the many data. Thus, a large screen which can show many data at once is needed. For these reasons, Xcode as IDE and iPad as Tablet PC are selected in this study.

Fig. 1 shows a main view of the software. Five kinds of data are used for a meal planning evaluation. Namely, data of nutrient, food groups, ingredients, patient data, and cooking method are needed for the evaluation of the meal planning. Nutrient has 14 items as shown in Table I. Food groups have 18 items or 6 items as shown in Table II. In this study, both of them are shown. Food groups and a kind of dishes are needed for Japanese food guide spinning top [7]. Basically, summations of each item of 
nutrient, food groups, and Japanese food guide spinning top are needed for meal planning evaluation. Additionally, the standard

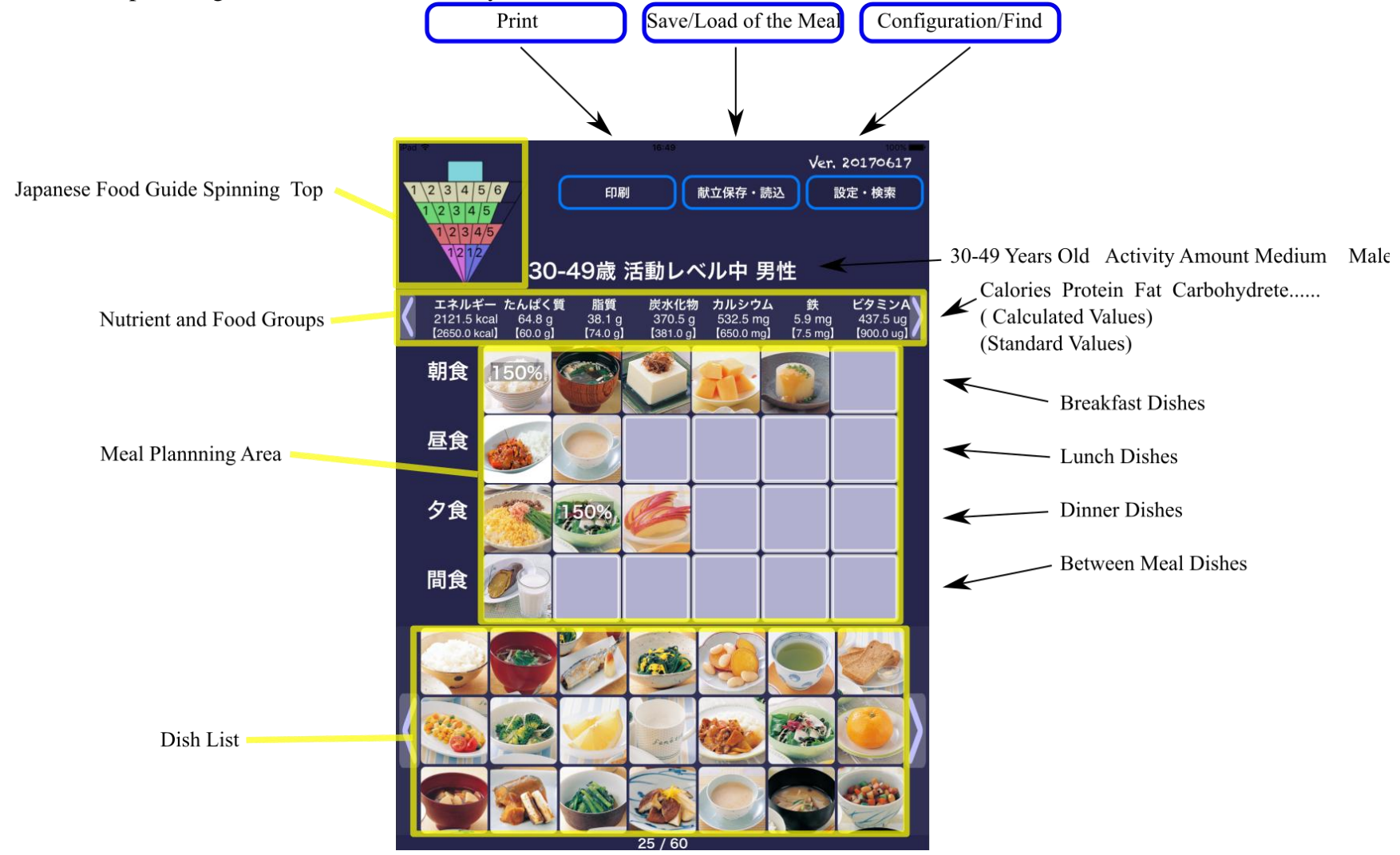

Fig. 1 Main View of Developed Software.

TABLE I

NUTRIENT ITEMS

\begin{tabular}{|l|l|l|}
\hline Calories & Protein & Fat \\
\hline Carbohydrate & Calcium & Iron \\
\hline Vitamin A & Vitamin D & Vitamin B1 \\
\hline Vitamin B2 & Vitamin C & $\begin{array}{l}\text { Saturated Fatty } \\
\text { Acids }\end{array}$ \\
\hline Dietary Fiber & Sodium Chloride & \\
\hline
\end{tabular}

T ABLE II

(A) EIGHTEEN FoOd GROUP ITEMS

\begin{tabular}{|l|l|}
\hline Grain & Tubers and roots \\
\hline Sugar & Nuts \\
\hline Green and Yellow Vegetables & Other Vegetables \\
\hline Fruits & mushrooms \\
\hline Seaweed & Bean \\
\hline seafood & Meat \\
\hline Egg & Dairy \\
\hline Oils and Fats (B) SiXFoOD GROUP ITEMS \\
\hline Performance beverage & Confectionaries \\
\hline \multicolumn{2}{|c|}{ Seasoning and Spice } \\
\hline Fish, Meat, Egg, Bean & Milk, small fish, seaweed \\
\hline Green and Yellow Vegetables & Other Vegetables and Fruits \\
\hline Grain, Tubers and roots, Sugar & Oils and Fats, Nuts \\
\hline
\end{tabular}

patient's age, gender, and activity amount is needed. Cooking method does not have amount. However, it is an important element for evaluating the meal planning, because time and effort can be estimated.

\section{A. Interface}

In a top left side, Japanese food guide spinning top is shown. By right side of the spinning top, three bottoms for print, save/load, and configuration/find are located. Patient's age, gender, and activity amount are shown under the three buttons. Next line shows a part of nutrient of a planning meal and these standard values of patient. This line can be swiped and remaining nutrient data and food groups data can be shown. Next four lines show a planning meal. First line shows a meal of breakfast. Similarly, second, third, and fourth lines show meals of lunch, dinner, and between meals. A square means one dish. Bottomthree lines show a dish list. A user can move a dish from the bottom list to the meal planning area by long tapping and dragging.

In this software, a dish is a unit for the meal planning. Namely, user does not need to consider about cooking detail, for instance, cooking method and time, amount and kinds of ingredients, and so on. Students can concentrate the combinations of dishes. It makes easy to plan a meal for students and give many trial and error experiences. This is one of the excellent points of this software.

By tapping a dish, a detail view of the dish is shown as shown in Fig. 2. In the top right side, print and return buttons are 
located. The top left side button is a favorite dish button. A name, a picture, ingredients, cooking method, nutrition, and food groups are shown. A user can check the detail information of the dish.

By tapping configuration/find button in the main view, configuration/find view are shown as shown in Fig. 3. The top right button is setting the configuration and returning to the main view. Pickers for age, activity amount, and gender are located. Many switches in the center means a configuration of the dish list in the main view. Namely, on state means showing the matched dishes. The left column shows cooking methods and the center and right columns show main ingredients. Lower side pickers mean the ascending/descending order of the selected nutrition item. Bottom buttons set a showing a dish name on the icon, showing favorite dishes, and showing selected dishes history.

By tapping save/load button in the main view, save/load view are shown. In this view, showing saved meal planning data, loading saved data, saving present data, and deleting saved data.

By tapping print button in the main view and the detail view, a printing dialog is shown. In order to use in offline state, meal planning data have to be saved on the tablet. Therefore, printed data is needed for checking or reporting the meal planning.

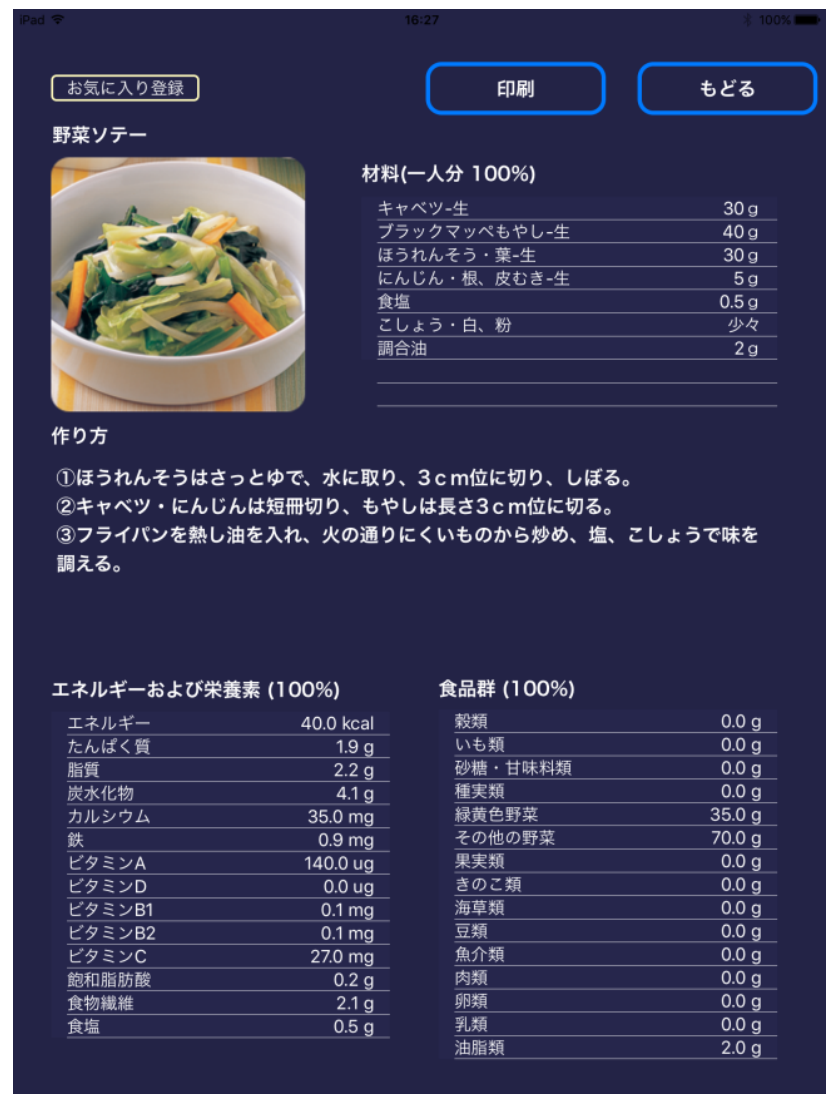

Fig. 2 Detail View of Developed Software.

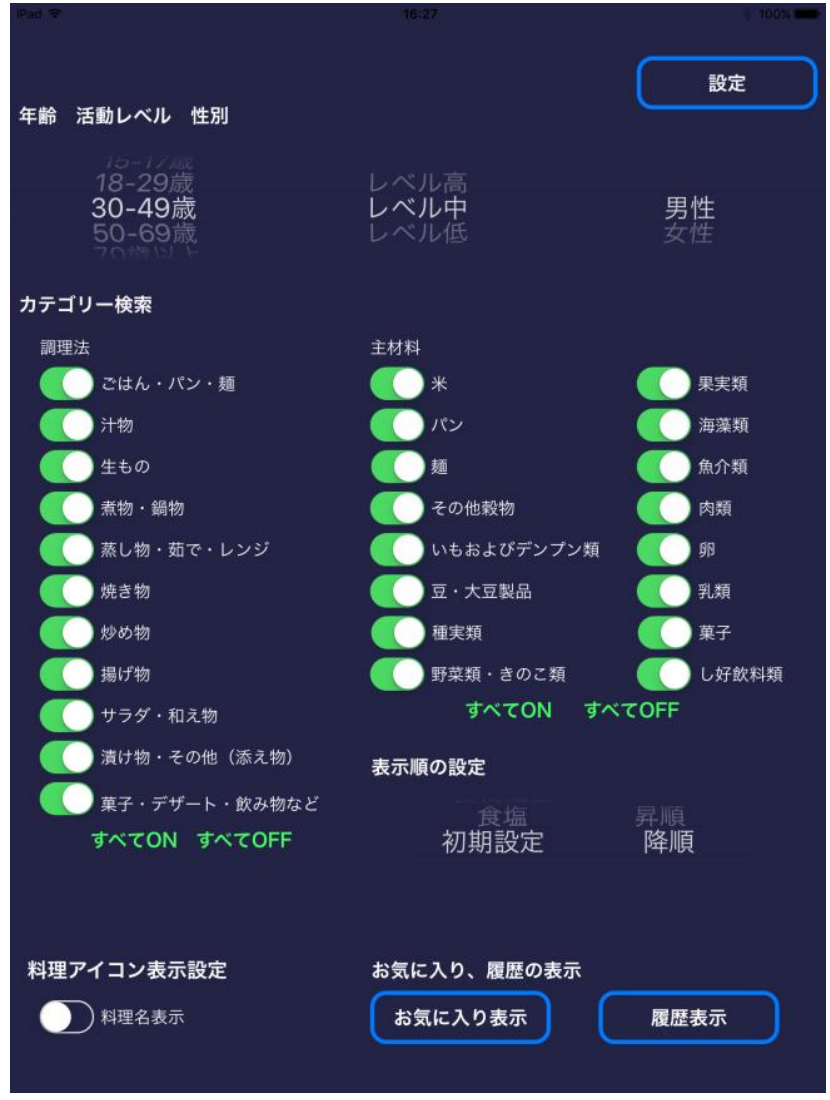

Fig. 3 Configuration/Find View of Developed Software.

T ABLE III

QUESTIONNAIRE RESULTS ABOUT DEVELOPED SOFTWARE

\begin{tabular}{|l|r|r|r|l|}
\hline & $\begin{array}{l}\text { Very } \\
\text { Hard } \\
(\%)\end{array}$ & \multicolumn{1}{|c|}{$\begin{array}{l}\text { Hard } \\
(\%)\end{array}$} & $\begin{array}{l}\text { Easy } \\
(\%)\end{array}$ & $\begin{array}{l}\text { Very } \\
\text { Easy } \\
(\%)\end{array}$ \\
\hline Configuration of a patient & 2.6 & 2.6 & 34.2 & 60.5 \\
\hline Choosing a dish & 2.6 & 2.6 & 31.6 & 63.2 \\
\hline Checking ingredients of a dish & 0 & 2.6 & 36.8 & 60.5 \\
\hline Deleting a selected dish & 0 & 2.6 & 26.3 & 71.1 \\
\hline Search function of a dish & 0 & 2.6 & 23.7 & 73.7 \\
\hline Checking Japanese food spinning top & 0 & 10.5 & 28.9 & 60.5 \\
\hline Finding a dish & 0 & 2.6 & 39.5 & 57.9 \\
\hline Changing amount of a dish & 0 & 2.6 & 44.7 & 52.6 \\
\hline Display ofnutrient values and standard values & 0 & 5.3 & 52.6 & 42.1 \\
\hline Display of printing and its contents & 0 & 7.9 & 52.6 & 39.5 \\
\hline Save function of a meal planning & 0 & 5.3 & 42.1 & 52.6 \\
\hline Load function of a meal planning & 0 & 2.6 & 39.5 & 57.9 \\
\hline Display of a dish name & 0 & 0 & 39.5 & 57.9 \\
\hline The number of dishes & 0 & 2.6 & 44.7 & 52.6 \\
\hline The kind of dishes & 0 & 2.6 & 42.1 & 55.3 \\
\hline
\end{tabular}

\section{TRAINING IN ClASS ROOMS}

Trainings of using the developed software have carried out in a clas sroom of Training Department of Adminis trative Dietitians, Faculty of Human Life Science, Shikoku University. Students were divided into two groups which consist of Group A and B. Group A students didn't use the application and use any other documents. Group B students used only the application. A questionnaire survey was carried out. By the questionnaire survey, following result are obtained from 78 students. 
Generally, when they plan a meal, many students refer to web sites $(80.3 \%)$ and cooking books $(63.2 \%)$ and some students refer to talk with family $(39.5 \%)$ and meals in their house $(35.5 \%)$.

When students decide a dish, "looks delicious" is most important point $(54.7 \%)$ and there are 5 differential points for deciding a dish between Group A and Group B. Group B is higher than Group A about a picture of a dish (A: 40.5\%, B:60.5\%), a nutrition of a dish (A:24.3\%, B: $55.3 \%)$, and balance among dishes of a meal (A:27.0\%, B:50.0\%).

When students plan meals, "the number of ingredients" and "the combination of dishes" are higher than $60 \%$. Differences of two groups are 5 items. Group B is higher than Group A about color, the number of ingredients, the number of dishes. On the other hand, Group A is higher than Group B about cooking time and cooking method.

Impressions of use about each views and functions of software were also asked. Table III shows the questionnaire results. As a result, almost students answered "Easy" or "Very Easy".

From these results, it is considered that developed application is useful for a meal planning with color and balancing nutrition.

\section{DISCUSSION}

This software is developed by basing on the book [8]. All data and pictures had been prepared. Calculation procedures of many items and showing items had been decided. Therefore, it had been considered that the main subject for developing the software was only technical skills. However, many other subjects had been obtained by revised many times by specialist and students. For instance, configuration/find view was changed many times. The favorite function was proposed by students. The print function was proposed by a specialist.

Our software development method is a kind of agile software development, because we discussed many times and two authors are specialists of diet and teachers of training of registered dietitians. Sometimes, the software specification was changed greatly. Some authors of specialists of information technology proposed suitable solutions for needs of diet specialis ts. Corroborations between specialists and information technology have many possibilities for information technology industry. In order to develop like this special software, it is considered that agile software development is suitable.

Some problems can be revealed by this development. One is a gap between two kinds of specialists. IT specialists who have many knowledge about IT don't have knowledge about diet. Especially, how to estimate a meal planning, calculation procedures, the number of items of food groups, and so on are common knowledge for dietitians. However, IT specialists didn't have these knowledges. Therefore, the source code of this software became spaghetti code. On the other hand, dietitians don't have knowledge about GUI, programming, database, and so on. Therefore, dietitian requested to revise the software only on an ad hoc basis. This gap is remaining even though this gap was reduced by agile software development.

Another problem is that this software is developed as offline system. In the classroom, it is not easy to connect many wifi devices. Additionally, using everywhere in a stable state is preferred for students. However, almost all tablet devices are utilized on online and many softwares are developed as online system. Developed system in this study also uses wifi for printing. If this software were developed as online system, more useful functions using the online state would be developed.

Our final goal is not only training of registered dietitian but also educating everyone for lifestyle disease prevention. Hence, smart phone version including android will be developed in the future. Additionally, data processing, save/load, and so on will be kept on cloud environment. By using cloud environment, data sharing and data analysis can be realized. This modification generates possibilities of corroborations between teachers and students, doctors and patients, students and patients, or so on. Furthermore, automatic evaluation function which is applied heuristic knowledge of teachers, adding new dish data function utilizing image processing and artificial intelligence, and so on will be implemented.

Information technology is payed attention from many other expert areas for progressing each area. Information technology is rapidly advancing step by step. In order to utilize information technology effectively, novel software development method which can be involving other experts as developers.

\section{CONCLUSION}

In this study, meal planning tablet software for training of registered dietitians was developed and used in the classroom. In order to have many trial and error experiences of meal planning, students of registered dietitians need to obtain the evaluations of their meal planning, immediately. Our software can obtain the evaluations and it is easy to use.

This software was developed by a corroboration between information technology and diet. Progressing information technology is very fast. New device, new system, new way to thinking, and so on are proposed. Understanding these new technologies is not easy for many people excepting experts of information technology. Thus, new method for apply new information technology to another field is needed.

As future works, smart phone version including android will be developed, automatic evaluation function which is applied heuristic knowledge of teachers, adding new dish data function utilizing image processing and artificial intelligence, and so on will be implemented.

\section{ACKNOWLEDGMENT}

This work was supported by JSPS KAKENHI Grant Number JP15K12366.

Data and pictures using this work was presented by KENPAKUSHA.

\section{REFERENCES}

[1] Kagawa Nutrition University, Eiyo-Pro Software ver.3.00, 2016

[2] Eatreat, Calcuation of diet, URL: https://eat-treat.jp/calculation

[3] Y. Yoshimura, Excel Eiyou-Kun Ver. 8.1, KENPAKUSHA, 2017

[4] Apple inc., iPad Official Web Site, URL: https://www.apple.com/ipad/ 
[5] Google, Android Official Web Site, URL: https://www.android.com/

[6] Apple inc., Xcode Official Web Site, URL: https://developer.apple.com/x

code/

[7] Ministry of agriculture, Forestry and Fisheries of Japan, Japanese Food Guide Spinning Top illustration in English (pdf). URL: http://www.maff.

go.jp/j/balance_guide/b_use/pdf/eng_reiari.pdf

[8] S. Watanabe, F. Teramoto, A. Tanaka, H. Kudo, S. Yanagisawa, Y. Matsuda, and K. Takahashi, "Eiyo Shokuji Ryoho Series (Nutrient diet Series)," 2009 KENP AKUSHA

[9] K. Ozaki, Y. Hosokawa, N. Suzuki, K. Takahashi, and S. Kawabata, "Menu Planning Support System -Development and Evaluation of Prototype Version-," Annual Bulletin of the Research Institute of Management and Information Science, Shikoku University vol.22, 2017 